INPLASY

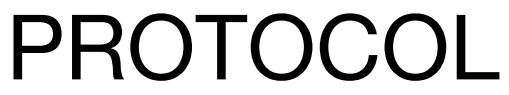

To cite: Kang et al. Effect of awake prone positioning in non-intubated COVID-19 patients with acute hypoxemic respiratory failure: a systematic review and meta-analysis. Inplasy protocol 2021110037. doi:

10.37766/inplasy2021.11.0037

Received: 11 November 2021

Published: 11 November 2021

Corresponding author:

Zhaohui Tong

tongzhaohuicy@sina.com

Author Affiliation:

Department of Respiratory and Critical Care Medicine, Beijing Institute of Respiratory Medicine, Beijing Chao-Yang Hospital, Capital Medical University, Beijing, 100020, China.

Support: Not applicable.

Review Stage at time of this submission: Piloting of the study selection process.

Conflicts of interest:

None declared.

\section{Effect of awake prone positioning in non-intubated COVID-19 patients with acute hypoxemic respiratory failure: a systematic review and meta-analysis}

\author{
Kang $\mathrm{H}^{1}$; Gu X2; Tong $\mathrm{Z}^{3}$.
}

Review question / Objective: This meta-analysis aims to assess the effect of awake prone positioning on intubation rate and mortality compared with standard care group in nonintubated patients with acute hypoxemic respiratory failure due to COVID-19.

Condition being studied: The awake prone positioning has been increasingly concerned during the COVID-19 pandemic. Some studies reported that awake prone positioning was effective in ameliorating blood oxygenation in non-intubated COVID-19 patients with acute respiratory failure. As for the intubation rate and mortality, the results of different studies are inconsistent. The updated Surviving Sepsis Campaign Guideline also stated that they were unable to issue a recommendation on the use of awake prone positioning in non-intubated COVID-19 adults because of the uncertainty about the effect on patient's important outcomes. Thus, we systematically reviewed the current trials and performed a meta-analysis to assess whether awake prone positioning can reduce the intubation rate and mortality of adults COVID-19 patients with acute hypoxemic respiratory failure than supine position.

INPLASY registration number: This protocol was registered with the International Platform of Registered Systematic Review and Meta-Analysis Protocols (INPLASY) on 11 November 2021 and was last updated on 11 November 2021 (registration number INPLASY2021110037).

\section{INTRODUCTION}

Review question / Objective: This metaanalysis aims to assess the effect of awake prone positioning on intubation rate and mortality compared with standard care group in non-intubated patients with acute hypoxemic respiratory failure due to COVID-19. 
Condition being studied: The awake prone positioning has been increasingly concerned during the COVID-19 pandemic. Some studies reported that awake prone positioning was effective in ameliorating blood oxygenation in non-intubated COVID-19 patients with acute respiratory failure. As for the intubation rate and mortality, the results of different studies are inconsistent. The updated Surviving Sepsis Campaign Guideline also stated that they were unable to issue a recommendation on the use of awake prone positioning in nonintubated COVID-19 adults because of the uncertainty about the effect on patient's important outcomes. Thus, we systematically reviewed the current trials and performed a meta-analysis to assess whether awake prone positioning can reduce the intubation rate and mortality of adults COVID-19 patients with acute hypoxemic respiratory failure than supine position.

\section{METHODS}

Participant or population: Adult ( $\geqslant 18$ years old) COVID-19 patients with acute hypoxemic respiratory failure or acute respiratory distress syndrome and in nonintubated state.

Intervention: Non-invasive respiratory support in the awake prone position.

Comparator: Non-invasive respiratory support not in the prone position.

Study designs to be included: Randomized controlled trials or observational studies.

Eligibility criteria: The inclusion criteria were as follows: 1) adult COVID-19 patients with acute hypoxemic respiratory failure or acute respiratory distress syndrome and in non-intubated state; 2) studies that compared experimental group using noninvasive respiratory support in the awake prone position with control group using non-invasive respiratory support not in the prone position; 3) studies included required outcomes and the data could be directly extracted or calculated; 4) randomized controlled trials or observational studies.

Information sources: Pubmed, Embase and Cochrane Library.

Main outcome(s): The primary outcomes included intubation rate and mortality.

Quality assessment / Risk of bias analysis: The risk of bias of the included RCTs were judged by the Cochrane Collaboration Risk of Bias tool. And we used the NewcastleOttawa scale to assess the risk of bias of the included observational cohort studies.

Strategy of data synthesis: All statistical analyses were conducted with Review Manager Version 5.4 and Stata version 16.0. Values for dichotomous outcomes were presented as the odds ratios (OR) with 95 $\%$ confidence intervals (Cl). And values for continuous outcomes were reported as the mean (standard deviation). The MantelHaenszel random effects model was used to analyze the results. The results of the meta-analysis were presented in the forest plots. Statistical heterogeneity between studies were assessed by $I^{2}$ testing. $I^{2}$ of more than $50 \%$ was regarded as moderateto-high heterogeneity. We used subgroup analyses and sensitivity analyses to explore the sources of heterogeneity. For the primary outcomes, subgroup analysis based on randomized controlled trials and observational studies was conducted to identify the potential sources of moderateto-high heterogeneity. Sensitivity analyses were also performed by sequentially omitting one study each time or excluded studies with intermediate or high risk of bias to find the potential influence. The publication bias was evaluated by the funnel plot and Egger tests when more than ten studies were included, and a $p$ value less than 0.05 was considered as the substantial publication bias.

Subgroup analysis: For the primary outcomes, subgroup analysis based on randomized controlled trials and observational studies was conducted to identify the potential sources of moderateto-high heterogeneity. 
Sensitivity analysis: Sensitivity analyses were performed by sequentially omitting one study each time or excluded studies with intermediate or high risk of bias to find the potential influence.

Country(ies) involved: China.

Keywords: COVID-19, acute hypoxemic respiratory failure, awake prone positioning, outcome, meta-analysis.

Contributions of each author:

Author 1 - Hanyujie Kang.

Author 2 - Xueqing Gu.

Author 3 - Zhaohui Tong. 